\title{
TRANSITIVITIES OF BETWEENNESS
}

\author{
BY \\ EVERETT PITCHER AND M. F. SMILEY
}

Introduction. The examination of the foundations of geometry which interested many prominent mathematicians about the turn of the century brought to light the importance of the fundamental notion of betweenness (see, for example $\left.\left({ }^{1}\right),[10,11]\right)$. This notion has suffered the treatment which modern mathematics metes out to all its concepts, namely, first an examination of the concept in a particular instance followed by wider and wider generalizations. The first part of this program for the concept of betweenness was carried through by Pasch, Huntington and Kline $[8,10]$. The simplicity of the concept permitted them to give an elegant and complete theory for the case of linear order. In the direction of generalizations $\left({ }^{2}\right), \mathrm{K}$. Menger and his students have been one of the most important influences in the study of betweenness in metric spaces $[9,3]$.

We purpose here to add to both phases of this program. The first part of our paper continues the analysis of Huntington and Kline into an examination of postulates involving five points; the second part deals mainly with a definition of betweenness in lattices which generalizes metric betweenness in metric lattices (see $[5,6]$ ). It is hoped that the five point transitivities may prove interesting and their analysis valuable. If we restrict our attention to the relation of betweenness in linear order such cannot be the case since four point properties are then sufficient to describe completely the betweenness relation. We feel that the results of the second part exhibit the properties of the betweenness relation as reflections of properties of the underlying space $\left({ }^{3}\right)$.

We shall use the notations of set theory which have become standard. In the second part we shall assume a knowledge of the fundamentals of both lattice theory and metric geometry. We refer the reader to the recent books Distance Geometry by L. M. Blumenthal [3] and Lattice Theory by Garrett Birkhoff [1]. We shall use the terminology and notation of these books in the second part.

Presented to the Society, May 2, 1941; received by the editors June 11, 1941.

(1) The numbers enclosed in brackets refer to the list of references at the end of the paper.

(2) The chordal systems recently introduced by W. Kaplan (Duke Mathematical Journal, vol. 7 (1940), pp. 165-167) are a generalization of linear order involving two triadic relations.

(3) The oft-quoted remark of K. Menger that Postulate B of Huntington and Kline should not be regarded as a property of betweenness but as a property of the underlying space $[9$, p. 79 ; 3, p. 36] indicates that it is easy to lose sight of this fact. 
TABLE OF CONTENTS

Part I. Transitivities of BetweEnNESS

1. Fundamental assumptions................................ 96

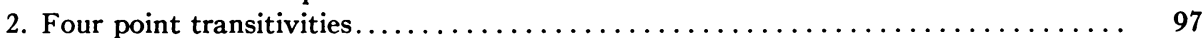

3. Weak transitivities on four points. $\ldots \ldots \ldots \ldots \ldots \ldots \ldots \ldots \ldots \ldots \ldots \ldots \ldots \ldots \ldots \ldots \ldots$

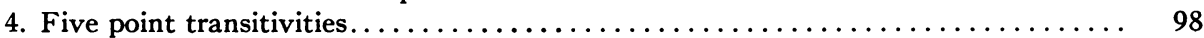

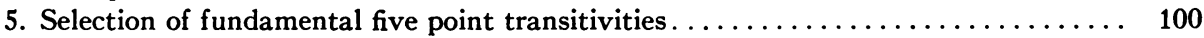

6. The logical relations among the fundamental strong transitivities $\ldots \ldots \ldots \ldots \ldots \ldots 1$

7. The examples in the existential theory ......................... 103

\section{Part II. Applications}

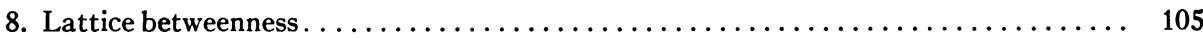

9. Interpretations of certain of the five point transitivities for lattice betweenness... 106

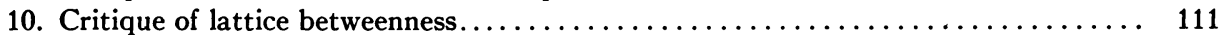

11. Betweenness in metric, semi metric, and metric ptolemaic spaces......... 113

\section{PART I}

We shall extend the discussion of an abstract relation of "betweenness" initiated by Pasch [10] and developed by Huntington and Kline [8] by relaxing some of the fundamental postulates of Huntington and Kline and by considering other possible postulates, particularly transitivities on five points.

1. Fundamental assumptions. We consider a set $K$ of points $a, b, c, d, x, \cdots$, and a triadic relation called betweenness, which holds (is positive) or fails (is negative) for each ordered triple of points, not necessarily distinct, in $K$. If the relation holds for the triple $a, b, c$, we write $a b c$, read as written or as " $b$ is between $a$ and $c$." We make the following assumptions throughout Part I.

$\alpha . a b c$ if and only if $c b a$ (symmetry in the end points).

$\beta$. $a b c$ and $a c b$ if and only if $b=c$ (closure).

Postulate $\alpha$ is Postulate A of Huntington and Kline [8]. Postulate $\beta$ is similar to their Postulate C. Postulates $\alpha$ and $\beta$ together imply the statements (1) and (2) below.

(1) $a b a$ if and only if $a=b$.

(2) Every two positive relations on three points (not necessarily distinct) are equivalent or inconsistent.

We do not assume that of an unordered triple of points one is between the other two (Postulate $\mathrm{B}$ of Huntington and Kline). In this respect our development will differ materially from theirs. This difference is essential because our interest lies in applications to lattices and metric spaces where B fails for very simple examples. We have replaced their Postulate $D$ which requires that the three points of a linear triple be pairwise distinct by $\beta$ because we wish our five point transitivities to specialize under identification of two points to four point transitivities. This change, though logically necessary, is essentially only a change in terminology. 
2. Four point transitivities. The statements about four points in which two positive relations of betweenness imply a third, which are theorems about linear order, and from which no hypothesis can be deleted leaving an equivalent statement, will be termed strong transitivities on four points. They are as follows.
$t_{1} . a b c \cdot a d b \rightarrow d b c$.
$t_{2} . a b c \cdot a d b \rightarrow a d c$.
$t_{3} . a b c \cdot b c d \cdot b \neq c \rightarrow a b d$.

These are postulates (3), (2), and (1), respectively, of Huntington and Kline and are completely discussed by them. We shall need the fact that the only implication among the three is: " $t_{1} \cdot t_{3} \rightarrow t_{2}$ " $[8$, p. 321].

3. Weak transitivities on four points. The statements concerning four points in which three distinct positive relations of betweenness imply a fourth, which are theorems about linear order, and from which no hypothesis may be deleted leaving an equivalent statement, will be termed weak transitivities on four points. They are as follows.

$\tau_{1} . a b c \cdot a d b \cdot a d c \rightarrow d b c$.

$\tau_{2} . a b c \cdot a d b \cdot d b c \rightarrow a d c$.

THEOREM 3.1. The statements $\tau_{1}$ and $\tau_{2}$ are the only weak transitivities on four points. The implications $t_{1} \rightarrow \tau_{1}, t_{2} \rightarrow \tau_{2}$ hold.

Proof. The second assertion is trivial. In order to prove the first assertion, we first observe that no two relations in hypothesis or conclusion of such a statement involve the same three letters by virtue of condition (2) of $\$ 1$. Next, there are four ways of selecting unordered triples from four letters. Let $a b c$ be the first hypothesis and $a, b, d$ be the letters in the second. Since $a, b, d$ or $b, c, d$ must occur in one hypothesis, it is always possible to achieve this situation by renaming the points. The second hypothesis is then one of the three, (1) $d a b$, (2) $a d b$, or (3) $a b d$. The third hypothesis is on the points (i) $b, c, d$ or (ii) $a, c, d$. We examine the possible relations on the three letters in one of the sets (i), (ii) with (1), (2), or (3) for consistency with linear order; we then examine the other one of the sets (i), (ii) for a conclusion of a theorem about linear order. In the eight cases we have:

Third hypothesis

$\begin{array}{lrll}(1) & \text { (i) } & b c d & a c d \\ (1) & (\text { ii) } & a c d & b c d \\ (2) & \text { (i) } & d b c & a d c \\ (2) & (\mathrm{ii}) & a d c & d b c \\ (3) & \text { (i) } & b c d & a c d \\ (3) & (\mathrm{i}) & b d c & a d c \\ (3) & (\mathrm{ii}) & a c d & b c d \\ (3) & \text { (ii) } & a d c & b d c\end{array}$


It is readily seen that these eight theorems reduce to two on suitable permutations of the letters of the letters of the hypotheses and conclusions. These two are $\tau_{1}$ and $\tau_{2}$. This completes the proof.

From this discussion of weak transitivities on four points it is apparent that an attempt at a weaker statement about four points with four or more hypotheses and one conclusion must contain two hypotheses or a hypothesis and a conclusion identical under $\alpha$ or a hypothesis or conclusion true under $\beta$.

4. Five point transitivities. The statements concerning five points in which three positive relations of betweenness imply a fourth, which are theorems about linear order, and from which no hypothesis can be deleted leaving an equivalent statement, will be termed strong transitivities on five points. They are as follows.

$$
\begin{aligned}
& T_{1} . \quad a b c \cdot a d b \cdot x d b \cdot b \neq d \rightarrow x d c . \\
& T_{2} . \quad a b c \cdot a d b \cdot b c x \cdot b \neq c \rightarrow d c x \text {. } \\
& T_{3} . \quad a b c \cdot a d b \cdot x c d \cdot c \neq d \rightarrow a c x \text {. } \\
& \text { T4. } a b c \cdot d a b \cdot x c d \quad \rightarrow a b x \text {. } \\
& \text { T5. } a b c \cdot a d c \cdot b x d \quad \rightarrow a x c \\
& T_{6} \cdot a b c \cdot a d b \cdot a c x \quad \rightarrow d c x \text {. } \\
& T_{7} \cdot a b c \cdot a b d \cdot c x d \quad \rightarrow a b x \text {. } \\
& T_{8} \cdot a b c \cdot d a b \cdot x c d \cdot a \neq b \rightarrow a c x . \\
& T_{9} \cdot a b c \cdot d a b \cdot x c d \cdot a \neq b \rightarrow b c x \text {. } \\
& T_{10} \cdot a b c \cdot a b d \cdot x b c \cdot a \neq b \cdot b \neq c \rightarrow x b d \text {. }
\end{aligned}
$$

$T_{11} \cdot a b c \cdot a d c \cdot x a b \cdot a \neq b \rightarrow x a d$.

$T_{12} \cdot a b c \cdot a d c \cdot x a b \cdot a \neq b \rightarrow x d c$.

$T_{13} \cdot a b c \cdot a d b \cdot x a b \cdot a \neq b \rightarrow x d c$.

$T_{14} \cdot a b c \cdot a d b \cdot x a c \quad \rightarrow x a d$.

$T_{15} \cdot a b c \cdot a d b \cdot x a c \quad \rightarrow x d b$.

$T_{16} \cdot a b c \cdot a d b \cdot x a c \quad \rightarrow x d c$.

$T_{17} \cdot a b c \cdot a d b \cdot a c x \quad \rightarrow a d x$.

$T_{18} \cdot a b c \cdot a d b \cdot a c x \rightarrow d b x$.

$T_{19} \cdot a b c \cdot a d b \cdot x a d \cdot a \neq d \rightarrow x a c$.

$T_{20} . a b c \cdot a d b \cdot x a d \cdot a \neq d \rightarrow x b c$.

$T_{21} \cdot a b c \cdot a d b \cdot x a d \cdot a \neq d \rightarrow x d c$.

$T_{22} \cdot a b c \cdot a d b \cdot b x c \quad \rightarrow a d x$.

$T_{23} \cdot a b c \cdot a d b \cdot b x c \quad \rightarrow d b x$.

$T_{24} \cdot a b c \cdot a d b \cdot b c x \cdot b \neq c \rightarrow a d x$.

$T_{25} \cdot a b c \cdot a d b \cdot b c x \cdot b \neq c \rightarrow d b x$.

$T_{26} \cdot a b c \cdot a d b \cdot b d x \cdot b \neq d \rightarrow x b c$.

$T_{27} \cdot a b c \cdot a d b \cdot x c d \cdot c \neq d \rightarrow a b x$.

$T_{28} \cdot a b c \cdot a d b \cdot x c d \cdot c \neq d \rightarrow a d x$.

$T_{29} \cdot a b c \cdot a d b \cdot x c d \quad \rightarrow d b x$. 


$$
\begin{array}{ll}
T_{30} \cdot a b c \cdot a d b \cdot x c d & \rightarrow b c x . \\
T_{31} \cdot a b c \cdot a d b \cdot c x d & \rightarrow a x c . \\
T_{32} \cdot a b c \cdot a d b \cdot c x d & \rightarrow a d x . \\
T_{33} \cdot a b c \cdot a d b \cdot c d x & \rightarrow x b c . \\
T_{34} \cdot a b c \cdot a d b \cdot c d x & \rightarrow x d b . \\
T_{35} \cdot a b c \cdot d a b \cdot a d x \cdot a \neq b \cdot a \neq d \rightarrow x a c . \\
T_{36} \cdot a b c \cdot d a b \cdot a d x \cdot a \neq b \cdot a \neq d \rightarrow x b c . \\
T_{37 \cdot} a b c \cdot d a b \cdot x c d \cdot a \neq b \rightarrow d a x . \\
T_{38 \cdot} a b c \cdot d a b \cdot x c d \cdot a \neq b \rightarrow d b x .
\end{array}
$$

THEOREM 4.1. The statements $T_{1}-T_{38}$ are a complete list of strong transitivities on five points.

Proof. In order to effect this enumeration we reason as follows. One letter in the three hypotheses must occur only once since there are nine places to be filled with five letters. We shall denote this letter (or one such if there are more) by $x$ and agree that it occurs in the third hypothesis. Then the first two hypotheses are on four letters with two letters in common. Letting $a b c$ be the first hypothesis and $d$ the remaining letter, we see that the second hypothesis must be on the letters (i) $a, c, d$ or (ii) $a, b, d$; the case $b, c, d$ reduces to $a, b, d$ on interchange of $a$ and $c$, which by virtue of $\alpha$ does not change $a b c$. Calling $a$ and $c$ in $a b c$ terminal and $b$ medial, we see that the letters common to the first and second hypotheses must fall under one of the following cases:

I. each letter terminal in both hypotheses;

II. one letter terminal in both; one letter medial once (say in the first) and terminal once;

III. one letter terminal in both; one medial in both;

IV. each letter terminal once and medial once.

Possible pairs of hypotheses to fill the first two places are then
A. $a b c$ and $a d c$,
C. $a b c$ and $a b d$,
B. $a b c$ and $a d b$,
D. $a b c$ and $d a b$.

On examination one will find that we have used the pairs I (i), II (ii), III (ii), and IV (ii). The pairs I (ii), II (i), III (i), and IV (i) are incompatible with linear order. With any of the pairs A, B, C, D we can use a third hypothesis on
(1) $a, b, x$,
(2) $a, c, x$,
(3) $a, d, x$,
(4) $b, c, x$,
(5) $b, d, x$,
(6) $c, d, x$.

Every letter which occurs only once in the hypotheses must occur in the conclusion. For, if we had a theorem about linear order for which this were false, 
we would obtain an equivalent one by dropping the hypothesis containing the letter; and we have agreed not to consider statements with this property.

In the six subcases (1)-(6) under A we can use a conclusion on

(1) or (4) $a, d, x ; b, d, x ; c, d, x$.

(2) $b, d, x$.

(3) $a, b, x ; b, c, x ; b, d, x$.

(5) $a, b, x ; a, c, x ; a, d, x ; b, c, x ; c, d, x$.

(6) $a, b, x ; b, c, x ; b, d, x$.

In the six subcases (1)-(6) under $\mathrm{B}, \mathrm{C}$, or $\mathrm{D}$ we can use a conclusion on

(1) $c, d, x$.

(2) or (4) $a, d, x ; b, d, x ; c, d, x$.

(3) or (5) $a, c, x ; b, c, x ; c, d, x$.

(6) $a, b, x ; a, c, x ; a, d, x ; b, c, x ; b, d, x$.

We proceed then to an examination of cases according to the following plan. With each pair A, B, C, D we inspect the three arrangements of the letters in the six cases of (4.1) to see whether they are consistent with linear order. With each consistent arrangement we inspect each of the three arrangements of letters in the corresponding set of (4.2) and (4.3) to determine whether a theorem in linear order is obtained. The work is shortened by examining each set of three hypotheses as we proceed to see whether it has already occurred under some permutation of the letters; this examination is facilitated by applying the classification scheme I-IV to the three pairs of hypotheses.

This procedure yields the transitivities $T_{1}-T_{38}$ though not in that order, and the proof is complete.

The reader will observe that the program initiated in the determination of the transitivities $\tau_{1}, \tau_{2}$, and $T_{1}-T_{38}$ could be extended to include transitivities with more hypotheses and more letters. We shall not do this.

5. Selection of fundamental five point transitivities. Each of the transitivities $T_{11}-T_{38}$ is equivalent to a combination of the transitivities $t_{1}, t_{2}, t_{3}$. We shall state and prove these facts in the following form

$T_{11}$. $\sim t_{1} \cdot t_{3}\left[b=c ; c=d . a b c, x a b\left(t_{3}\right) x a c, a d c\left(t_{1}\right) x a d.\right]$

We mean that $T_{11}$ is equivalent to $t_{1}$ and $t_{3}$; that $t_{1}$ is proved by identifying $b$ and $c$; that $t_{3}$ is proved by identifying $c$ and $d$; that $T_{11}$ is proved from $t_{1}$ and $t_{3}$ by applying $t_{3}$ to $a b c$ and $x a b$ to obtain $x a c$, and by applying $t_{1}$ to $x a c$ and $a d c$ to obtain xad. We use $\alpha$ and $\beta$ freely without explicit reference. Whenever we use $t_{3}$ the letters common to the two hypotheses are distinct as required. 
$T_{12}$. $t_{2} \cdot t_{3}\left[b=c ; a=d . a b c, x a b\left(t_{3}\right) x a c, a d c\left(t_{2}\right) x d c.\right]$

$T_{13}$. $t_{2} \cdot t_{3}\left[b=c ; a=d . a b c, a d b\left(t_{2}\right) a d c ; a b c, x a b\left(t_{3}\right) x a c, a d c\left(t_{2}\right) x d c.\right]$

$T_{14}$. $t_{1}\left[b=c . a b c, x a c\left(t_{1}\right) x a b, a d b\left(t_{1}\right) x a d.\right]$

$T_{15}$. $t_{1} \cdot t_{2}\left[a=d ; b=c . a b c, x a c\left(t_{1}\right) x a b, a d b\left(t_{2}\right) x d b.\right]$

$T_{16} \sim t_{2}\left[a=x . a b c, a d b\left(t_{2}\right) a d c, x a c\left(t_{2}\right) x d c.\right]$

$T_{17}$. $t_{2}\left[c=x . a b c, a d b\left(t_{2}\right) a d c, a c x\left(t_{2}\right) a d x.\right]$

$T_{18}$. . $t_{1} \cdot t_{2}\left[b=c ; a=d . a b c, a c x\left(t_{2}\right) a b x, a d b\left(t_{1}\right) d b x.\right]$

$T_{19} . \sim t_{3}\left[b=c . a d b, x a d\left(t_{3}\right) x a b, a b c\left(t_{3}\right) x a c.\right]$

$T_{20}$. $t_{3}\left[b=d . a d b, x a d\left(t_{3}\right) x a b, a b c\left(t_{3}\right) x b c.\right]$

$T_{21}$. $\sim t_{2} \cdot t_{3}\left[a=x ; b=c . a b c, a d b\left(t_{2}\right) a d c, x a d\left(t_{3}\right) x d c.\right]$

$T_{22}$. $\sim t_{1} \cdot t_{2}\left[b=d ; c=x . a b c, b x c\left(t_{1}\right) a b x, a d b\left(t_{2}\right) a d x.\right]$

$T_{23}$. . $t_{1}\left[a=d . a b c, a d b\left(t_{1}\right) d b c, b x c\left(t_{1}\right) d b x.\right]$

$T_{24}$. . $t_{2} \cdot t_{3}\left[c=x ; b=d . a b c, b c x\left(t_{3}\right) a b x, a d b\left(t_{2}\right) a d x.\right]$

$T_{25}$..$t_{1} \cdot t_{3}\left[c=x ; a=d . a b c, b c x\left(t_{3}\right) a b x, a d b\left(t_{1}\right) d b x.\right]$

$T_{26}$. $\sim t_{1} \cdot t_{3}\left[d=x ; a=d . a b c, a d b\left(t_{1}\right) d b c, b d x\left(t_{3}\right) x b c.\right]$

$T_{27}$. . $t_{2} \cdot t_{3}\left[a=d ; b=c . a b c, a d b\left(t_{2}\right) a d c, d c x\left(t_{3}\right) a c x, a b c\left(t_{2}\right) a b x.\right]$

$T_{28}$. . $t_{2} \cdot t_{3}\left[c=x ; b=c . a b c, a d b\left(t_{2}\right) a d c, x c d\left(t_{3}\right) a d x.\right]$

$T_{29}$. . $t_{1} \cdot t_{2}\left[c=x ; a=d . a b c, a d b\left(t_{1}\right) d b c, x c d\left(t_{2}\right) d b x.\right]$

$T_{30}$. . $t_{1}\left[a=d . a b c, a d b\left(t_{1}\right) d b c, x c d\left(t_{1}\right) b c x.\right]$

$T_{31}$. . $t_{2}\left[b=c . a b c, a d b\left(t_{2}\right) a d c, c x d\left(t_{2}\right) a x c.\right]$

$T_{32} . \sim t_{1} \cdot t_{2}\left[b=c ; c=x . a b c, a d b\left(t_{2}\right) a d c, c x d\left(t_{1}\right) a d x.\right]$

$T_{33}$. . $t_{1} \cdot t_{2}\left[d=x ; a=d . a b c, a d b\left(t_{1}\right) d b c, c d x\left(t_{2}\right) x b c.\right]$

$T_{34}$. $t_{1}\left[a=d . a b c, a d b\left(t_{1}\right) d b c, c d x\left(t_{1}\right) x d b.\right]$

$T_{35}$. $t_{3}\left[b=c . a b c, d a b\left(t_{3}\right) c a d, a d x\left(t_{3}\right) x a c.\right]$

$T_{36}$. . $t_{3}\left[d=x . d a b, a d x\left(t_{3}\right) x a b, a b c\left(t_{3}\right) x b c.\right]$

$T_{37} \sim t_{2} \cdot t_{3}\left[b=c ; c=x . a b c, d a b\left(t_{3}\right) d a c, x c d\left(t_{2}\right) d a x.\right]$

$T_{38}$. $t_{2} \cdot t_{3}\left[a=d ; c=x . a b c, d a b\left(t_{3}\right) d b c, x c d\left(t_{2}\right) d b x.\right]$

We summarize the results of this section in the following theorem.

THEOREM 5. Each of the transitivities $T_{11}-T_{38}$ is equivalent to a combination of the transitivities $t_{1}, t_{2}$, and $t_{3}$.

6. The logical relations among the fundamental strong transitivities. None of the transitivities $T_{1}-T_{10}$ is equivalent to a combination of the transitivities $t_{1}, t_{2}, t_{3}$. We shall devote this section and the following one to a proof of this fact. In addition we shall construct the essentials of a complete existential theory of $t_{1}-t_{3}, T_{1}-T_{10}$. The basic implications are given in this section. We use the notation explained in $\$ 5$.

$t_{1} \cdot t_{3} \rightarrow T_{1} \rightarrow t_{2} \cdot t_{3}\left[a b c, a d b\left(t_{1}\right) d b c, x d b\left(t_{3}\right) x d c . a=x ; a=d.\right]$

$t_{1} \cdot t_{3} \rightarrow T_{2} \rightarrow t_{3}\left[a b c, a d b\left(t_{2}\right) a d c ; a b c, b c x\left(t_{3}\right) a c x, a d c\left(t_{1}\right) d c x . a=d.\right]$

$t_{2} \cdot t_{3} \rightarrow T_{3} \rightarrow t_{3}\left[a b c, a d b\left(t_{2}\right) a d c, x c d\left(t_{3}\right) a c x . b=c.\right]$ 


$$
\begin{aligned}
t_{1} \cdot t_{3} \rightarrow & T_{4} \rightarrow t_{1} \cdot t_{2}\left[a b c, d a b\left(t_{3}\right) d b c, x c d\left(t_{2}\right) d b x, d a b\left(t_{1}\right) a b x . b=c ; a=d .\right] \\
& T_{5} \rightarrow t_{2}[a=b .] \\
t_{1} \cdot t_{2} \rightarrow & T_{6} \rightarrow t_{1}\left[a b c, a d b\left(t_{2}\right) a d c, a c x\left(t_{1}\right) d c x . b=c .\right] \\
& T_{7} \rightarrow t_{1}[b=d .] \\
t_{1} \cdot t_{3} \rightarrow & T_{8} \rightarrow t_{1}\left[a b c, d a b\left(t_{3}\right) d a c, x c d\left(t_{1}\right) a c x . b=c .\right] \\
t_{1} \cdot t_{3} \rightarrow & T_{9} \rightarrow t_{1}\left[a b c, d a b\left(t_{3}\right) d b c, x c d\left(t_{1}\right) b c x . a=d .\right]
\end{aligned}
$$

It is apparent that when two letters of a statement $T_{1}-T_{10}$ are identified, the resulting statement is either a tautology or is equivalent to one of the statements $t_{1}, t_{2}, t_{3}, \tau_{1}$ or $\tau_{2}$. We may see that we cannot thus obtain either $\tau_{1}$ or $\tau_{2}$ as follows. Notice that the hypotheses of both $\tau_{1}$ and $\tau_{2}$ contain one letter three times and three letters twice and that the conclusion of each is on these latter three letters. Suppose that an identification of two letters leads to $\tau_{1}$ or $\tau_{2}$. Then $x$ must be identified with some letter because it occurs only once in the hypotheses of each of $T_{1}-T_{10}$. Since $x$ always appears in the conclusions of $T_{1}-T_{10}$, the letter to be identified with $x$ can occur only once in the hypotheses. It must then also occur in the conclusion along with $x$. By virtue of $\beta$ the conclusion is then either vacuous or implies still further identification. This contradicts our assumption that one of the statements $\tau_{1}$ or $\tau_{2}$ appears on identifying two letters.

The above list of implications includes all nontautological results obtained by identifying two letters. This fact will be useful in simplifying the examination of the table of examples to be given in $\$ 7$.

In the proofs of the following implications, the results of the preceding implications are used.

$$
\begin{gathered}
T_{8} \rightarrow T_{6}\left[a b c, a d b\left(t_{1}\right) d b c, a d b, a c x, d \neq b\left(T_{8}\right) d c x .\right. \\
\text { If } \left.d=b \text { then } a b c, a c x\left(t_{1}\right) d c x .\right] \\
T_{8} \rightarrow T_{9}\left[a b c, d a b, x c d, a \neq b\left(T_{8}\right) a c x, a b c\left(t_{1}\right) b c x .\right]
\end{gathered}
$$

$T_{4} \cdot T_{9} \rightarrow T_{8}\left[a b c, d a b, x c d\left(T_{4}\right) a b x ; a b c, d a b, x c d, a \neq b\left(T_{9}\right) b c x ; a b x, b c x\right.$ $\left.\left(t_{2}\right) a c x.\right]$

$T_{7} \cdot T_{9} \rightarrow T_{8}\left[a b c, d a b, x c d, a \neq b\left(T_{9}\right) b c x, d a b, x c d\left(T_{7}\right) x c a.\right]$

$t_{2} \cdot T_{8} \rightarrow T_{4}\left[a b c, d a b, x c d, a \neq b\left(T_{8}\right) a c x, a b c\left(t_{2}\right) a b x\right.$. If $a=b, T_{4}$ is true.]

$t_{1} \cdot T_{7} \rightarrow T_{6}\left[a b c, a d b\left(t_{1}\right) d b c ; a b c, a c x\left(t_{1}\right) b c x, a c x, a d b\left(T_{7}\right) d c x\right.$. $]$

$t_{2} \cdot t_{3} \cdot T_{10} \rightarrow T_{1}\left[a b c, a d b\left(t_{2}\right) a d c, a d b, x d b, a \neq d, b \neq d\left(T_{10}\right) x d c\right.$.

$$
\text { If } a=d, a b c, x a b\left(t_{3}\right) x d c \text {.] }
$$

We shall devote the next section to the proof of the following theorem.

THEOREM 6. The implications listed in this section are the only ones holding among the statements $t_{1}-t_{3}, T_{1}-T_{10}$.

REMARK. It seems to be worth mentioning that $t_{1} \cdot t_{2} \cdot t_{3} \rightarrow T_{1}, T_{2}, T_{3}, T_{4}, T_{6}$, $T_{8}, T_{9}$; but that $t_{1} \cdot t_{2} \cdot t_{3}$ does not imply $T_{5}, T_{7}$, or $T_{10}$ (for proof see $\S 7$ ). We are of the opinion that the interest of a five point transitivity varies inversely 
as its logical intimacy with $t_{1}, t_{2}$, and $t_{3}$. Viewed in this light, $T_{10}$ is surely the most interesting-but we still lack a "concrete" interpretation for it.

7. The examples in the existential theory. We shall complete the existential theory begun in $\S 6$. We have attempted to make our list of examples as simple as possible through the use of composite examples. No attempt has been made to make the number of points in each example the least possible $[14$, p. 250].

The following elementary examples will be used in the table which concludes this section. In each of the examples the positive relations are those listed together with the ones which follow from $\alpha$ and $\beta$. In the first four examples the class $K$ consists of four distinct points, while in the remaining examples $K$ consists of five distinct points. Certain of these examples are merely the statement of the hypotheses of one of the transitivities. We indicate this by giving the example the same number as the transitivity, replacing $t$ by $k, \tau$ by $\kappa$ and $T$ by $K$.
k1. $a b c a d b$.
k3. $a b c d a b$.
$\kappa 1$. $a b c$ adb $a d c$.
$\kappa 2$. $a b c \quad a d b \quad d b c$.
K3. $a b c$ adb $x c d$.
K4. $a b c$ dab $x c d$.
K5. $a b c$ adc $b x d$.
K7. $a b c$ abd $c x d$.
K10. $a b c$ abd $x b c$.
E1. $a b c \quad d a b$ xcd $a b x$.
E2. $a b c$ dab $x c d \quad b c x$.
E3. $a b c$ dab $x c d$ acx $b c x$.
E4. $a b c$ adb $a c x \quad d b c \quad b c x$.
E5. $a b c$ adb $b c x$ adc $a c x \quad a b x \quad a d x$.
E6. $a b c \quad a d b \quad x d b \quad a d c$.
E7. $a b c$ adb $b c x \quad a b x \quad a c x$.

The following table of examples completes our existential theory. In entry 4 we take as the space $K$ the points $a, b, c, d, x, a^{\prime}, b^{\prime}, c^{\prime}, d^{\prime}, x^{\prime}$ with the positive relations of example $K 5$ on the points $a, b, c, d, x$, the positive relations of example $K 7$ on the points $a^{\prime}, b^{\prime}, c^{\prime}, d^{\prime}, x^{\prime}$, and the other positive relations required by $\beta$. Each case in which the example column contains more than one entry is to be treated similarly. We have made no column for $T_{10}$. It will be found to hold in each of our examples except 32-35, where it must fail because of the implication $t_{2} \cdot t_{3} \cdot T_{10} \rightarrow T_{1}$. To secure the example corresponding to those listed in which $T_{10}$ fails we simply adjoin $K 10$ to the example listed. 


\begin{tabular}{|c|c|c|c|c|c|c|c|c|c|c|c|c|c|}
\hline & $t_{1}$ & $t_{2}$ & $t_{3}$ & $T_{1}$ & $T_{2}$ & $T_{3}$ & $T_{4}$ & $T_{5}$ & $T_{6}$ & $T_{7}$ & $T_{8}$ & $T_{9}$ & Example \\
\hline $\begin{array}{l}1 . \\
2 . \\
3 . \\
4 .\end{array}$ & + & + & + & + & + & + & + & $\begin{array}{l}+ \\
+ \\
-\end{array}$ & + & $\begin{array}{l}+ \\
+ \\
-\end{array}$ & + & + & $\begin{array}{l}\text { Linear order } \\
K 7 \\
K 5 \\
K 5, K 7\end{array}$ \\
\hline $\begin{array}{c}5 . \\
6 . \\
7 . \\
8 . \\
9 . \\
10 . \\
11 . \\
12-18 .\end{array}$ & + & + & - & - & - & - & $\begin{array}{l}+ \\
+ \\
+ \\
+ \\
- \\
- \\
-\end{array}$ & $\begin{array}{l}+ \\
+ \\
+ \\
+ \\
+ \\
+ \\
+ \\
-\end{array}$ & + & $\begin{array}{l}+ \\
+ \\
- \\
- \\
+ \\
- \\
*\end{array}$ & $\begin{array}{l}+ \\
\overline{+} \\
- \\
- \\
- \\
-\end{array}$ & $\begin{array}{l}+ \\
\overline{+} \\
- \\
- \\
+ \\
-\end{array}$ & $\begin{array}{l}k 3 \\
E 1 \\
k 3, K 7 \\
E 1, K 7 \\
K 4 \\
E 2 . \\
K 4, K 7 \\
\text { as in } 5-11 \\
\text { with } K 5\end{array}$ \\
\hline $\begin{array}{l}19 . \\
20 . \\
21 . \\
22 . \\
23 . \\
24 . \\
25 .\end{array}$ & + & - & - & - & - & - & - & - & $\begin{array}{l}+ \\
+ \\
+ \\
+ \\
+ \\
- \\
-\end{array}$ & $\begin{array}{l}+ \\
+ \\
- \\
- \\
- \\
-\end{array}$ & $\begin{array}{l}+ \\
+ \\
+ \\
- \\
- \\
-\end{array}$ & $\begin{array}{l}+ \\
- \\
+ \\
+ \\
+ \\
+\end{array}$ & $\begin{array}{l}E 3 \\
K 4, \kappa 2 \\
E 3, K 7 \\
E 2, \kappa 2 \\
K 4, K 7, \kappa 2 \\
E 4 \\
E 4, K 4\end{array}$ \\
\hline $\begin{array}{l}26 . \\
27 . \\
28 . \\
29 . \\
30 . \\
31 . \\
32 . \\
33 .\end{array}$ & - & + & + & $\begin{array}{l}+ \\
+ \\
+ \\
+ \\
- \\
- \\
-\end{array}$ & $\begin{array}{l}+ \\
+ \\
- \\
- \\
+ \\
+ \\
-\end{array}$ & + & - & $\begin{array}{l}+ \\
\bar{t} \\
- \\
+ \\
- \\
+\end{array}$ & - & - & - & - & $\begin{array}{l}\kappa 1 \\
\kappa 1, K 5 \\
E 5 \\
E 5, K 5 \\
E 6 \\
E 6, K 5 \\
E 5, E 6 \\
E 5, E 6, K 5\end{array}$ \\
\hline $\begin{array}{l}34 . \\
35 .\end{array}$ & - & + & - & - & - & - & - & $\begin{array}{l}+ \\
-\end{array}$ & - & - & - & - & $\begin{array}{l}\kappa 1, k 3 \\
\kappa 1, k 3, K 5\end{array}$ \\
\hline $\begin{array}{l}36 . \\
37 . \\
38 . \\
39 .\end{array}$ & - & - & + & - & $\begin{array}{l}+ \\
+ \\
-\end{array}$ & $\begin{array}{l}+ \\
+ \\
-\end{array}$ & - & - & - & - & - & - & $\begin{array}{l}k 1 \\
K 3 \\
E 7 \\
E 7, K 3\end{array}$ \\
\hline 40. & - & - & - & - & - & - & - & - & - & - & - & - & $k 1, k 3$ \\
\hline
\end{tabular}

* In these places arrange + and - signs as in the entries 5-11.

\section{PART II}

We shall devote the remainder of this paper to the study of a generalization of metric betweenness in metric lattices, and to the application of the transitivities of Part I both to this relation and to the relation of betweenness in semi metric, metric, and metric ptolemaic spaces. 
8. Lattice betweenness. Glivenko $[5,6]$ proved that in a metric lattice an element $b$ is (metrically) between the elements $a$ and $c$ if and only if

$$
(a \cap b) \cup(b \cap c)=b=(a \cup b) \cap(b \cup c) .
$$

This condition does not involve the metric and we take it as our definition $\left.{ }^{4}\right)$ of betweenness in an arbitrary lattice $L$. When $b$ is between $a$ and $c$ we shall frequently write simply $a b c$. We shall need the simple and fundamental properties of this relation given in the following two lemmas $\left(^{5}\right)$.

LEMMA 8.1. If $L$ is a lattice and $a, b, c \in L$, then

(1) the inequalities $a \leqq b \leqq c$ imply that the relation abc holds;

(2) the relation abc implies that $a \cap c \leqq b \leqq a \cup c$;

(3) both $a \cap c$ and $a \cup c$ are between $a$ and $c$.

Proof. (1) If $a \leqq b \leqq c$, then $(a \cap b) \cup(b \cap c)=a \cup b=b=b \cap c=(a \cup b)$ $\cap(b \cup c)$. By our definition, $a b c$; and $(1)$ is proved.

(2) If $a b c$, then $b \cap(a \cap c)=(a \cup b) \cap(b \cup c) \cap(a \cap c)=a \cap c$. It follows that $a \cap c \leqq b$. Dually, $b \leqq a \cup c$. This proves (2).

(3) Note that $(a \cap(a \cup c)) \cup((a \cup c) \cap c)=a \cup c$, and also that $(a \cup(a \cup c))$ $\cap((a \cup c) \cup c)=a \cup c$. By definition, $a \cup c$ is between $a$ and $c$. Dually, $a \cap c$ is between $a$ and $c$. This proves (3).

Lemma 8.2. If $L$ is a lattice then its betweenness relation satisfies $\alpha$ and $\beta$.

Proof. $(\alpha)$ This is an immediate consequence of the commutativity of the operations $a \cap b$ and $a \cup b$ in lattices.

$(\beta)$ Let $L$ be a lattice containing elements $a, b, c$ for which the relations $a b c$ and $a c b$ hold. We then have $b=(a \cup b) \cap(b \cup c)$ and $c=(a \cup c) \cap(c \cup b)$, and hence $b \cap c=(a \cup b) \cap(b \cup c) \cap(c \cup a)$. Consequently,

$$
\begin{aligned}
& b \cap c \geqq(a \cup b) \cap(b \cup c) \cap b=b \geqq b \cap c, \\
& b \cap c \geqq c \cap(b \cup c) \cap(c \cup a)=c \geqq b \cap c .
\end{aligned}
$$

It follows that $b \cap c=b=c$. To prove the converse we must show that $a a c$ is valid in lattices for every pair of elements $a, c$. It is easily seen that $(a \cap a) \cup(a \cap c)=a \cup(a \cap c)=a$. Using duality we see by the definition that the relation $a a c$ holds. The proof is complete.

In addition to these fundamental properties, we now show that lattice betweenness possesses the five point transitivity $T_{6}$.

THEOREM 8.1. If $L$ is a lattice then its betweenness relation satisfies the transitivity $T_{6}$.

(4) G. Birkhoff [1, p. 9] also gives a definition of betweenness which applies to partially ordered sets and which has all the transitivities of Part I.

(5) We may also note that $a b c$ holds if and only if $a \bigcap_{c} \leqq b \leqq a \cup_{c}$ and $(a, b, c) D$ (see J. von Neuman, Continuous Geometries, Princeton Lecture Notes, 1936-1937; and Lemma 10.1 below). 
Proof. Let $L$ be a lattice and consider elements $a, b, c, d, x \in L$ for which the relations $a b c, a d b$, and $a c x$ hold. We wish to show that $d c x$ is true. We prove first that $(d \cap c) \cup(c \cap x)=c$. Notice that $c=(a \cap c) \cup(c \cap x)$; and, by Lemma 8.1 (2), that $a \cap c \leqq b$, and $a \cap b \leqq d$. It follows that $a \cap c \leqq a \cap b \leqq d$. We obtain

$$
\begin{aligned}
(d \cap c) \cup(c \cap x) & =(d \cap((a \cap c) \cup(c \cap x))) \cup(c \cap x) \\
& \geqq(d \cap a \cap c) \cup(d \cap c \cap x) \cup(c \cap x) \\
& \geqq(a \cap c) \cup(d \cap c \cap x) \cup(c \cap x) \\
& \geqq(a \cap c) \cup(c \cap x) \\
& \geqq c \geqq(d \cap c) \cup(c \cap x) .
\end{aligned}
$$

Consequently, $(d \cap c) \cup(c \cap x)=c$. Dually, $(d \cup c) \cap(c \cup x)=c$. By definition, we have $d c x$. The proof is complete.

COROLlaRY. The transitivities $t_{1}$ and $\tau_{1}$ are valid for the betweenness relation in every lattice.

Proof. This is a trivial result of the implications $T_{6} \rightarrow t_{1} \rightarrow \tau_{1}$.

9. Interpretations of certain of the five point transitivities for lattice betweenness. Glivenko [5] showed that a metric lattice is distributive if and only if its (metric) betweenness relation has the transitivity which we have labeled $T_{5}$. We shall extend this result to lattice betweenness in this section. We shall also prove that both $T_{4}$ and $T_{7} \cdot t_{2}$ are equivalent to the distributive law; that each of the transitivities $t_{2}$ and $\tau_{2}$ is equivalent to the modular law; and that each one of the postulates $t_{3}, T_{1}, T_{2}$, and $T_{3}$ holds if and only if the lattice is linearly ordered. The remaining transitivities do not seem to have important lattice-theoretic interpretations. We shall verify that each of them fails in the Boolean algebra of eight elements.

Our first theorem gives the interpretation of the transitivity $t_{2}$.

THEOREM 9.1. A lattice $L$ is modular if and only if its betweenness relation satisfies the transitivity $t_{2}$.

Proof. Consider first a modular lattice $L$ containing elements $a, b, c, d$ for which the relations $a b c$ and $a d b$ hold. We wish to establish the relation $a d c$. Note that $(a \cap b) \cup(b \cap c)=b,(a \cap d) \cup(d \cap b)=d$, and, by Lemma 8.1 (2), that $a \cap c \leqq b$, and $a \cap b j \leqq d$. We then obtain $d=(a \cap d) \cup(d \cap b)=(a \cap d) \cup$ $(d \cap((a \cap b) \cup(b \cap c)))$. Using the modular law, since $a \cap b \leqq d$, we find that

$$
\begin{aligned}
d & =(a \cap d) \cup(a \cap b) \cup(d \cap b \cap c) \\
& =(a \cap d) \cup(d \cap b \cap c) \\
& \leqq(a \cap d) \cup(d \cap c) \leqq d .
\end{aligned}
$$

Hence $d=(a \cap d) \cup(d \cap c)$. Dually, $d=(a \cup d) \cap(d \cup c)$. Consequently, the relation $a d c$ is valid. Thus the modular law implies the transitivity $t_{2}$. Conversely, 
the transitivity $t_{2}$ implies the modular law. To see this, let $L$ be a lattice whose betweenness satisfies $t_{2}$. If $L$ is non-modular it must contain the simplest non-modular lattice of five elements shown in Figure 9.1 as a sublattice.

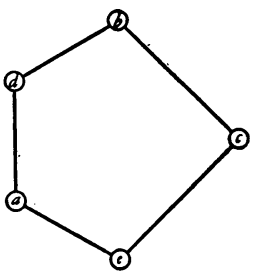

FIG. 9.1

Note that, by Lemma 8.1 (3), the relation $a b c$ holds in $L$ since $b=a \cup c$; and that the relation $a d b$ holds in $L$ by Lemma 8.1 (1). But if the relation $a d c$ is true, then $(a \cap d) \cup(d \cap c)=d$. However, we see from Figure 9.1 that $(a \cap d) \cup(d \cap c)=a \neq d$. Thus the transitivity $t_{2}$ fails in $L$. This is contrary to our hypothesis. It follows that the lattice $L$ is modular. The proof is complete.

A similar result holds for the transitivity $\tau_{2}$.

THEOREM 9.2. If $L$ is a lattice, then its betweenness relation satisfies the transitivity $\tau_{2}$ if and only if $L$ is modular.

Proof. If $L$ is a modular lattice, it is clear from the implication $t_{2} \rightarrow \tau_{2}$ and Theorem 9.1 that $\tau_{2}$ is valid for the betweenness of $L$. On the other hand, if $\tau_{2}$ holds then the lattice must be modular. Otherwise a sublattice such as we have pictured in Figure 9.1 exists. In it we have shown, in the proof of Theorem 9.1, that the relations $a b c$ and $a d b$ are true and that the relation $a d c$ is false. But the relation $d b c$ also holds in the lattice of Figure 9.1, since $b=d \cup c$. Hence the hypotheses of the transivity $\tau_{2}$ hold in this sublattice (and therefore also in the lattice itself), but its conclusion fails. This is contrary to the assumption that the transivity $\tau_{2}$ holds. Thus the transitivity $\tau_{2}$ implies that the modular law is valid. The proof is complete.

We pass now to a discussion of the transitivity $T_{5}$. Our next lemma, on the road to establishing the equivalence of $T_{5}$ and the distributive law, gives a relation between Duthie's segments $\left(^{6}\right)$ and our betweenness.

LEMma 9.1. If $L$ is a lattice then it is distributive if and only if for every triple $a, b, c \in L$ the inequalities $a \cap c \leqq b \leqq a \cup c$ imply that the relation abc holds.

Proof. Consider a lattice $L$ in which the implication of our lemma holds. We establish the modular law for $L$ first. Consider three elements $a, b, c \in L$ with $a \leqq c$. Since $a \cap b \leqq c \cap(a \cup b) \leqq a \cup b$, our hypothesis yields that $c \cap(a \cup b)$ is between $a$ and $b$. Whence we have

(6) Duthie defines a segment of a lattice $L$ between two elements $a, b \in L$ as the set of all $x \in L$ satisfying $a \bigcap b \leqq x \leqq a \cup b$. Our lemma has also been proved by him [4]. 


$$
\begin{aligned}
c \cap(a \cup b) & =(a \cap c \cap(a \cup b)) \cup(c \cap(a \cup b) \cap b) \\
& =(a \cap c) \cup(c \cap b)=a \cup(b \cap c),
\end{aligned}
$$

which is the modular law. Now consider elements $u, v, w \in L$. Note that $(u \cap w) \leqq(u \cap w) \cup(v \cap(u \cup w)) \leqq(u \cup w)$. By hypothesis we obtain that $z \equiv(u \cap w) \cup(v \cap(u \cup w))$ is between $u$ and $w$. An easy application of the modular law reduces the conditions that this relation hold to the equations

$$
(u \cap v) \cup(v \cap w) \cup(w \cap u)=z=(u \cup v) \cap(v \cup w) \cap(w \cup u) .
$$

But this last identity characterizes distributive lattices $[1$, p. 74]. Conversely, if $L$ is distributive and $a, b, c$ are three elements of $L$ for which $a \cap c \leqq b \leqq a \cup c$, then $(a \cap b) \cup(b \cap c)=b \cap(a \cup c)=b$, and dually. Thus the relation $a b c$ holds. This completes the proof.

We continue with the proof that $T_{5}$ is equivalent to the distributive law.

THEOREM 9.3. If $L$ is a lattice, then its betweenness relation has the transitivity $T_{5}$ if and only if $L$ is distributive.

Proof. Consider a lattice $L$ whose betweenness relation satisfies $T_{5}$. By Lemma 9.1, $L$ will be distributive provided the relation $a b c$ holds for every triple $a, b, c \in L$ such that $a \cap c \leqq b \leqq a \cup c$. Hence consider elements $a, b, c \in L$ for which $a \cap c \leqq b \leqq a \cup c$. By Lemma 8.1 (2), $b$ is between $a \cap c$ and $a \cup c$. By Lemma 8.1 (3), we know that both $a \cap c$ and $a \cup c$ are between $a$ and $c$. Application of the transitivity $T_{5}$ then yields the fact that $b$ is between $a$ and $c$. Thus the validity of the transitivity $T_{5}$ implies the distributive law by Lemma 9.1. Conversely, if $L$ is distributive and the relations $a b c$, $a d c$, and $b x d$ hold for elements $a, b, c, d, x \in L$, then, using Lemma 8.1 (2), we obtain

$$
a \cap c \leqq b \cap d \leqq x \leqq b \cup d \leqq a \cup c .
$$

Since $L$ is distributive, it then follows from Lemma 9.1 that $b$ is between $a$ and $c$. Hence the distributive law implies that the transitivity $T_{5}$ holds in $L$. This completes the proof.

Still another form of the distributive law is provided by the postulate $T_{4}$, while $T_{7}$ is equivalent to the distributive law in modular lattices. The next three theorems will show this.

THEOREM 9:4. If $L$ is a distributive lattice, then its betweenness relation has the transitivities $T_{4}$ and $T_{7}$.

Proof. Let $L$ be a distributive lattice. We prove first that $T_{4}$ holds for the betweenness of $L$. Consider five elements $a, b, c, d, x \in L$ for which the relations $a b c, d a b$, and $x c d$ hold. We wish to prove that the relation $a b x$ is valid. By Lemma 9.1 it is sufficient to show that $a \cap x \leqq b \leqq a \cup x$. Lemma 8.1 (2) yields that $a \leqq b \cup d$, and that $x \cap d \leqq c$. Hence we find that 


$$
a \cap x \leqq x \cap(b \cup d)=(x \cap b) \cup(x \cap d) \leqq(x \cap b) \cup c .
$$

Combining with $a$ we have

$$
a \cap x \leqq a \cap((x \cap b) \cup c)=(a \cap x \cap b) \cup(a \cap c) \leqq(a \cap b) \cup(b \cap c) .
$$

But $(a \cap b) \cup(b \cap c)=b$, since the relation $a b c$ holds. It follows that $a \cap x \leqq b$. Dually, $a \cup x \geqq b$. By Lemma 9.1 and the fact that $L$ is distributive we then know that the relation $a b x$ is valid. Thus the transitivity $T_{4}$ is valid in distributive lattices.

To prove that $T_{7}$ holds for the betweenness relation of a distributive lattice $L$, consider five elements $a, b, c, d, x \in L$ for which the relations $a b c, a b d$, and $c x d$ hold. We wish to show that we have the relation $a b x$. By Lemma 8.1 (2), we know that $a \cap c \leqq b, a \cap d \leqq b$, and that $x \leqq c \cup d$. Combining the last inequality with $a$, we find that $a \cap x \leqq a \cap(c \cup d)=(a \cap c) \cup(a \cap d) \leqq b$. Dually, $a \cup x \geqq b$. It follows from Lemma 9.1 that the relation $a b x$ is true. Thus $T_{7}$ is valid in distributive lattices. The proof is complete.

THEOREM 9.5. If $L$ is a lattice whose betweenness relation has the transitivity $T_{4}$, then $L$ is distributive.

Proof. The implication $T_{4} \rightarrow t_{2}$, proved in $\S 6$, together with the result of Theorem 9.1 shows that if $T_{4}$ holds for lattice betweenness in a lattice $L$ then $L$ is modular. It is well known $[1$, p. 75$]$ that every modular non-dis-

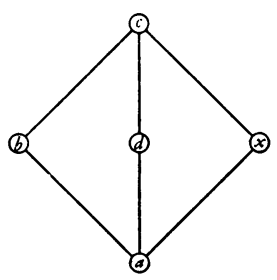

FIG. 9.2

tributive lattice contains a copy of the simplest modular non-distributive lattice shown in Figure 9.2 as a sublattice. Thus if $T_{4}$ were to hold for lattice betweenness in a non-distributive lattice $L$ it would hold in the lattice of Figure 9.2. In this lattice we have the relations $a b c, d a b$, and $x c d$ since $a<b<c$, $a=b \cap d$, and $c=d \cup x$. But $a b x$ would require that $(a \cap b) \cup(b \cap x)=b$, while actually $(a \cap b) \cup(b \cap x)=a \cup a=a \neq b$. Thus $T_{4}$ fails in this lattice. It follows that the transitivity $T_{4}$ for lattice betweenness implies that the lattice is distributive. The proof is complete.

THEOREM 9.6. If $L$ is a modular lattice whose betweenness relation satisfies the transitivity $T_{7}$ then $L$ is distributive.

Proof. Since $L$ is modular it can fail to be distributive only if it has a sublattice of the type shown in Figure 9.2. If we reletter the elements of this 
lattice, putting $a^{\prime}=b, b^{\prime}=a, c^{\prime}=x, d^{\prime}=d$, and $x^{\prime}=c$, we may verify easily that the relations $a^{\prime} b^{\prime} c^{\prime}, a^{\prime} b^{\prime} d^{\prime}$, and $c^{\prime} x^{\prime} d^{\prime}$ hold since $b^{\prime}=a^{\prime} \cap c^{\prime}, b^{\prime}=a^{\prime} \cap d^{\prime}$, and $x^{\prime}=c^{\prime} \cup d^{\prime}$. If $T_{7}$ held we should have $\left(a^{\prime} \cup b^{\prime}\right) \cap\left(b^{\prime} \cup x^{\prime}\right)=b^{\prime}$, while in fact

$$
\left(a^{\prime} \cup b^{\prime}\right) \cap\left(b^{\prime} \cup x^{\prime}\right)=a^{\prime} \cap x^{\prime}=a^{\prime} \neq b^{\prime} .
$$

Thus $T_{7}$ fails for $L$. It follows that a modular lattice $L$ cannot fail to be distributive when $T_{7}$ holds. The proof is complete.

REMARK. An examination of the lattice of Figure 9.1 will show that the result of Theorem 9.6 cannot be extended to non-modular lattices.

Our next theorem discusses the transitivities $T_{1}, T_{2}, T_{3}$, and $t_{3}$.

THEOREM 9.7. If $L$ is a lattice then its betweenness relation has one of the transitivities $T_{1}, T_{2}, T_{3}, t_{3}$ if and only if $L$ is linearly ordered.

Proof. Since the transitivities cited obviously hold in a linear order and since each of them implies $t_{3}$, it will suffice to show that the betweenness relation of a lattice satisfies $t_{3}$ only if the lattice is a linear order. Hence let $L$ be a lattice whose betweenness relation satisfies $t_{3}$. Consider two elements $a, b \in L$. Suppose that none of the relations $a<b, a>b, a=b$ holds. Then clearly $a \cup b \neq a$ and $a \cap b \neq a$. Note that $a \cap b<a<a \cup b$. By Lemma 8.1 (1), we find that $a$ is between $a \cup b$ and $a \cap b$. By Lemma 8.1 (3), $a \cup b$ is between $a$ and $b$. The transitivity $t_{3}$ then yields the fact that $a$ is between $a \cap b$ and $b$. It follows that

$$
a=(b \cap a) \cup(a \cap(a \cap b))=(a \cap b) \cup(a \cap b)=a \cap b,
$$

contrary to the fact that $a \cap b \neq a$. Thus, if $t_{3}$ holds for the betweenness of $L$ no pair of elements of $L$ can be incomparable. This means that $L$ is linearly ordered. The proof is complete.

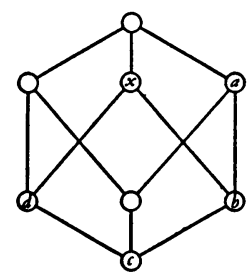

FIG. 9.3

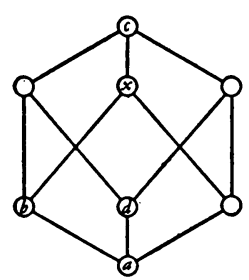

FIG. 9.4

We now show that each of the remaining transitivities, namely, $T_{8}, T_{9}$, and $T_{10}$, fail to hold in the Boolean algebra of eight elements. To see that $T_{10}$ fails note that in Figure 9.3 we have $a b c, a b d, x b c$, and $b x d$ since $a<b<c$, $a \cap d<b<a \cup d, c<b<x$, and $x=b \cup d$; but if $x b d$ also held then $\beta$ would require that $x=b$. Figure 9.4 provides a counterexample for $T_{8}$ and $T_{9}$. Using Lemma 8.1, we see that we have $a b c, d a b$, and $x c d$ since $a<b<c, a=d \cap b$, 
and $c=d \cup x$. But $b c x$ is false since $b \cup x=x$, which does not contain $c$; and $a c x$ is false since $a \cup x=x$.

Let us summarize the results of this section and the preceding one in a theorem.

THEOREM 9.8. If $L$ is a lattice then its betweenness relation has the transitivities $T_{6}, t_{1}$, and $\tau_{1}$; it has each of the transitivities $t_{2}$ and $\tau_{2}$ if and only if $L$ is modular; it has each of the transitivities $T_{4}$ and $T_{5}$ if and only if $L$ is distributive; it has the transitivity $T_{7}$ if and only if $L$ is distributive provided that $L$ is modular; and it has each of the transitivities $t_{3}, T_{1}, T_{2}$, and $T_{3}$ if and only if $L$ is linearly ordered.

10. Critique of lattice betweenness. A. Wald found a set of properties of metric betweenness which characterize this relation in metric spaces [13]. We shall devote this section to a proof of an analogous result for lattice betweenness. The algebraic structure of lattices permits a slight economy in that we may characterize our relation of lattice betweenness in the particular lattice considered, while Wald found it necessary to consider a relation $R$ defined in every metric space.

The present form of this section is due in large measure to suggestions of W. R. Transue. He, together with one of us, applies the result in a study of transitivities of betweenness in metric lattices and their generalizations.

Our result takes the following form.

THEOREM 10.1. If $L$ is a lattice and $R$ is a triadic relation defined for all ordered $\left({ }^{7}\right)$ triples of elements of $L$, then $R$ is lattice betweenness provided that the following conditions hold.

(i) $R$ satisfies the postulates $(\alpha)$ and $(\beta)$.

(ii) $R$ satisfies the transitivity $t_{1}$.

(iii) If $a \leqq b \leqq c$, then $(a, b, c) R$.

(iv) The relations $(a, a \cup c, c) R,(a, a \cap c, c) R$ hold for every $a, c \in L$.

(v) If the relation abc holds, then in the sublattice generated by $a, b, c$ the transitivity $t_{2}$ holds for $R$.

The properties (i)-(iv) have already been established for lattice betweenness in Lemmas 8.1 and 8.2 and in the corollary to Theorem 8.1. The following lemma justifies the assumption (v).

LEMMA 10.1. If $L$ is a lattice and the relation abc holds for three elements $a, b, c \in L$, then the sublattice generated by $a, b, c$ is distributive.

Proof. We shall prove, in fact, that the free lattice $[1$, p. 22] generated by three elements $a, b, c$ for which the relation $a b c$ is assumed is given in

(7) This word "ordered" refers, of course, to the fundamental metamathematical notion of "ordered" set. This should not be confused with the order relation in the lattice $L$. 
Figure 10.1. To prove this, let $a, b, c$ be three elements which generate a lat-

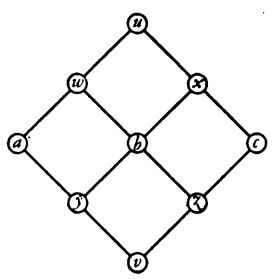

FIG. 10.1

tice in which the relation $a b c$ holds. We note first that we must then have $b=(a \cup b) \cap(b \cup c)$. Hence we must also have

$$
\begin{aligned}
((a \cup b) \cap c) \cup b) & =((a \cup b) \cap c) \cup((a \cup b) \cap(b \cup c)) \\
& =(a \cup b) \cap(b \cup c)=b .
\end{aligned}
$$

It follows that $b \geqq(a \cup b) \cap c$. Consequently, $b \cap c \geqq(a \cup b) \cap c \geqq b \cap c$, and hence $b \cap c=(a \cup b) \cap c$. Using this result we see that $(b \cap c) \cup(a \cap c)=$ $(c \cap(a \cup b)) \cup(a \cap c)=c \cap(a \cup b)=b \cap c$. Interchange of $a$ and $c$ in these results and their duals justifies Figure 10.1. It is obvious that the lattice of Figure 10.1 is distributive since it is the product [1, p. 13 and p. 76] of two chains of three elements. The proof is complete.

Proof of Theorem 10.1. Consider a lattice $L$ and a triadic relation $R$ defined for all ordered triples of elements of $L$ which satisfies the conditions (i) $-(\mathrm{v})$ of Theorem 10.1. We prove first that the relation $(a, b, c) R$ implies the relation $a b c$. For this implication we need only the conditions (i)-(iv). Consider three elements $a, b, c \in L$ for which the relation $(a, b, c) R$ holds. By (iv) we have $(a, a \cup b, b) R$ and (ii) then gives $(a \cup b, b, c) R$. Again (iv) gives $(b, b \cup c, c) R$ and (ii) yields $(a \cup b, b, b \cup c) R$. Note that $b \leqq(a \cup b) \cap(b \cup c)$ $\leqq a \cup b$, and apply (iii) to obtain $(b,(a \cup b) \cap(b \cup c), a \cup b) R$. Combining this last relation with $(a \cup b, b, b \cup c) R$ and using (ii) we find that $(b \cup c, b,(a \cup b) \cap(b \cup c)) R$. But since $b \leqq(a \cup b) \cap(b \cup c) \leqq b \cup c$, (iii) gives $(b,(a \cup b) \cap(b \cup c), b \cup c) R$. Using (i) we then obtain $b=(a \cup b) \cap(b \cup c)$. By duality, $b=(a \cap b) \cup(b \cap c)$, and we conclude that the relation $a b c$ holds. Thus the relation $(a, b, c) R$ implies the relation $a b c$.

We prove next that the relation $a b c$ implies the relation $(a, b, c) R$. For this implication we do not use the condition (ii). In the proof we shall omit explicit reference to our use of the condition (i). Let $a, b, c$ be three elements of $L$ for which the relation $a b c$ holds. By Lemma 8.1 (2) we have $a \leqq a \cup b \leqq a \cup b \cup c$ $=a \cup c$, and the relations $(a, a \cup b \cup c, c) R$ and $(a, a \cup b, a \cup b \cup c) R$ then follow from (iv) and (iii). Condition (v) then gives $(a, a \cup b, c) R$. Note that $c \leqq b \cup c \leqq a \cup b \cup c$. The relations $(c, b \cup c, a \cup b \cup c) R$ and $(c, a \cup b \cup c, a \cup b) R$ then follow from (iii) and (iv). Applying (v) we obtain $(c, b \cup c, a \cup b) R$. Since $a b c$ holds we have $b=(a \cup b) \cap(b \cup c)$, and (iv) then gives $(b \cup c, b, a \cup b) R$. 
Condition ( $\mathrm{v})$ then yields $(c, b, a \cup b) R$. Combining this last relation with $(c, a \cup b, a) R$ and using (v) again we find $(a, b, c) R$. Thus the relation $a b c$ implies the relation $(a, b, c) R$.

Combining the results of the preceding two paragraphs we find that the relation $R$ holds if and only if lattice betweenness holds, that is, $R$ is the lattice betweenness of $L$. The proof of Theorem 10.1 is complete.

REMARK. It seems unfortunate that our theorem requires the condition (v). That it is necessary to make some such assumption may be seen by considering the lattice of Figure 10.1. In this lattice let $R$ be the same as lattice betweenness except that the relation $(a, b, c) R$ does not hold. If we could prove $(a, b, c) R$ from the assumptions (i)-(iv) of Theorem 10.1, then we should have to obtain this result from condition (ii) since the conclusions of (i), (ii), and (iii) cannot apply to a triple $(d, e, f)$ with both $d$ and $e$ and $f$ and $e$ not comparable. To obtain $(a, b, c) R$ from (ii) would require hypotheses of the form $(d, b, c) R,(d, a, b) R$ or of the form $(d, b, a) R,(d, c, b) R$. But these sets cannot hold in our example, since if we have $(d, a, b) R$ and $(d, b, c) R$, then $d \neq a$, and $d \cap b \leqq a \leqq d \cup b$. It follows that $d \cup b=w$ or $u$ and hence that $d \cap b=b$, contrary to $d \cap b \leqq a$. The other set of hypotheses may be treated likewise by interchanging $a$ and $c$. It is possible to give alternatives for the condition ( $v$ ) but we shall not consider them here.

11. Betweenness in metric, semi metric, and metric ptolemaic spaces. In a metric space with distance function $\delta$ one says $[3$, p. 38] that $q$ is between the points $p$ and $r$ in case $\delta(p, q)+\delta(q, r)=\delta(p, r)$ and $p \neq q \neq r$. It is evident that this relation fails to satisfy our condition $\beta$. We suggest that it should be modified so as to satisfy $\beta$ by deleting the condition $p \neq q \neq r$ which requires that the points $p, q, r$ be pairwise distinct. We shall do this and shall write $p q r$ for the modified relation, reserving the locution " $q$ is between $p$ and $r$ " for the usual relation. K. Menger [9] established the transitivities $t_{1}$ and $t_{2}$ for metric betweenness. His famous example of a "railroad" space [9, p. 80 ] was constructed to prove that the transitivity $T_{5}$ may fail in metric spaces. For the case of a semi metric space [3, p. 38] O. Taussky found that the weak transitivity $\tau_{1}$ holds for the analogue of metric betweenness. Examples of semi metric spaces are easily given in which $\tau_{2}$ fails.

There has recently been some interest in spaces which are metric and ptolemaic $[12,2]$, that is, metric spaces in which the three products of the lengths of opposite sides of every quadrilateral are the sides of some triangle in the euclidean plane. For such spaces L. M. Blumenthal [2] established the transitivity $t_{3}$. Thus in metric ptolemaic spaces we have immediately the properties $T_{1}-T_{4}, T_{8}$, and $T_{9}$. It is interesting that $T_{5}$ also holds in such spaces. We may see this as follows $\left(^{8}\right)$. Let $a, b, c, d, x$ be five points of a metric ptolemaic space which satisfy the relations $a b c, a d c$, and $b x d$. Using the ptole-

(8) Professor Blumenthal has also noted this fact in a letter to one of us. We are indebted to him for a stimulating correspondence during the preparation of this paper. 
maic inequality $\left({ }^{9}\right)$ we obtain $a x \cdot b d \leqq a b \cdot x d+a d \cdot x b$ and $c x \cdot b d \leqq b c \cdot x d+d c \cdot x b$. Adding these inequalities we find

$$
b d(a x+c x) \leqq x d(a b+b c)+x b(a d+d c)=x d \cdot a c+x b \cdot a c=b d \cdot a c .
$$

If $b=d$, then by (1) of $\S 1, b=d=x$, and the relation $a x c$ is implied by the relation $a b c$. If $b \neq d$, then $a x+c x=a c$ from (11.1) and the triangle inequality, and the relation $a x c$ is true. As an example of the use of the relation $a b c$ instead of " $b$ is between $a$ and $c$," let us give the proof of $T_{5}$ for the second relation. It will suffice to prove that $a \neq x \neq c$. Suppose that $a=x$. By hypothesis, $a$ is then between $b$ and $d$. The transitivity $t_{3}$ then gives $(a \neq b !)$ that $a$ is between $d$ and $c$, which contradicts $d$ between $a$ and $c$. If $x=c$, then by hypothesis $c$ is between $a$ and $d$. The transitivity $t_{3}$ then gives $(b \neq c !)$ that $c$ is between $a$ and $d$, which contradicts $d$ between $a$ and $c$.

None of the remaining five points transitivities, namely, $T_{6}, T_{7}$, and $T_{10}$, holds in every metric ptolemaic space. This may be shown by examples of spaces consisting of five points.

\section{REFERENCES}

1. Garrett Birkhoff, Lattice Theory, American Mathematical Society Colloquium Publications, vol. 25, 1940.

2. L. M. Blumenthal, Betweenness in metric ptolemaic spaces, Bulletin of the American Mathematical Society, abstract 47-1-66.

3. - Distance Geometries, The University of Missouri Studies, vol. 13, no. 2 (1938).

4. W. D. Duthie, Segments of ordered sets, these Transactions, vol. 51 (1942), pp. 1-14.

5. V. Glivenko, Contributions a l'étude des systèmes de choses normées, American Journal of Mathematics, vol. 59 (1937), pp. 941-956.

6. - Géométrie des systèmes de choses normées, American Journal of Mathematics, vol. 58 (1936), pp. 799-828.

7. E. V. Huntington, $A$ new set of postulates for betweenness with proof of complete independence, these Transactions, vol. 26 (1924), pp. 257-282.

8. E. V. Huntington and J. R. Kline, Sets of independent postulates for betweenness, these Transactions, vol. 18 (1917), pp. 301-325.

9. K. Menger, Untersuchungen über allgemeine Metrik, Mathematische Annalen, vol. 100 (1928), pp. 75-163.

10. Pasch-Dehn, Grundlagen der Geometrie, Berlin, 1926.

11. Gilbert de B. Robinson, The Foundations of Geometry, Mathematical Expositions, Toronto, no. 1, 1940.

12. I. J. Schoenberg, Metric arcs of vanishing Menger curvature, Annals of Mathematics, (2), vol. 41 (1940), pp. 715-726.

13. A. Wald, Axiomatik des Zwischenbegriffes in metrischen Räume, Mathematischen Annalen, vol. 104, (1930-1931), pp. 476-484.

14. W. E. van de Walle, On the complete independence of the postulates for betweenness, these Transactions, vol. 24 (1926), pp. 249-256.

LEHIGH UNIVERSITY, Bethlehem, Pa.

( $\left.{ }^{9}\right)$ For convenience we now write the distance between two points $a$ and $b$ of the metric space simply as $a b$. 